\title{
Sampling in Complex and Harmonic Analysis
}

\author{
Joaquim Bruna
}

\begin{abstract}
This is a survey article on uniqueness, sampling and interpolation problems in complex analysis. Most of these problems are motivated by applications of great practical importance in signal analysis and data transmission, but they also admit other mathematical formulations relating them to fundamental questions about existence of good bases in function spaces. This circle of ideas in complex analysis has experimented in recent years a notorious revitalization, mostly because of its connections with analogous problems in time-frequency and wavelet analysis, some of which will be discussed as well.
\end{abstract}

\section{Sampling and Interpolation for Band Limited Functions}

In this paper we will be talking mostly about sampling, and the most well-known sampling result is the one dealing with band-limited functions, known as the Shannon-Whitaker theorem (or Kotelnikov theorem in Russia). A $\tau$-band-limited function is one with finite energy, i.e. $\int|f(t)|^{2} d t<+\infty$, whose Fourier transform $\hat{f}(\zeta)$

$$
\hat{f}(\zeta)=\frac{1}{\sqrt{2 \pi}} \int_{-\infty}^{+\infty} f(t) e^{-i t \zeta} d t
$$

vanishes for $|\zeta|>\tau$. Since $f(t)=\frac{1}{\sqrt{2 \pi}} \int_{-\tau}^{+\tau} \hat{f}(\zeta) e^{i t \zeta} d \zeta$, one may think $f$ as being an (infinite) linear combination of sine and cosine functions with frequencies $|\zeta|<\tau$. We denote by $B_{\tau}^{2}$ the space of $\tau$-band limited functions; note that the Fourier transform is an isometry between $B_{\tau}^{2}$ and $L^{2}(-\tau, \tau)$.

The KSW sampling theorem states that the general form of such a function is given by the so called cardinal series

$$
f(t)=\sum_{k=-\infty}^{+\infty} a_{k} \frac{\sin (\tau t-\pi k)}{\tau t-\pi k}
$$

with $\sum_{k}\left|a_{k}\right|^{2}<+\infty$. Note that $a_{k}=f\left(\frac{k \pi}{\tau}\right)$ and, in fact,

$$
\sum_{k}\left|a_{k}\right|^{2}=\frac{\tau}{\pi} \int_{-\infty}^{+\infty}|f(t)|^{2} d t
$$


There are two aspects in this statement which is convenient to state separately. First, every $f \in B_{\tau}^{2}$ is completely recovered from its samples $f\left(\frac{k \pi}{\tau}\right)$ in a stable way, that is, for $f, g \in B_{\tau}^{2}$

$$
\int_{-\infty}^{+\infty}|f(t)-g(t)|^{2} d t=\frac{\pi}{\tau} \sum_{k}\left|f\left(\frac{k \pi}{\tau}\right)-g\left(\frac{k \pi}{\tau}\right)\right|^{2} .
$$

This means that a small error in the collection of samples $\left\{f\left(\frac{k \pi}{\tau}\right)\right\}_{k \in Z}$ will produce a small error in the construction. Secondly, any square-summable sequence of numbers appear in this way, no other restriction appears.

Both aspects constitute the theoretical basis for the transition from analog signals $f(t)$ to discrete sequences $\left\{a_{k}\right\}_{k \in Z}$, and is of great practical importance in communications and data transmission in general.

The theorem is just a restatement of the fact that the exponential system $\left\{\frac{1}{\sqrt{2 \tau}} e^{i \frac{\pi}{\tau} k \zeta}\right\}_{k \in Z}$ is an orthonormal basis of $L^{2}(-\tau, \tau)$. In the expansion of $\hat{f}(\zeta)$ in this basis, the coefficients are

$$
\left\langle\hat{f}, \frac{1}{\sqrt{2 \tau}} e^{i \frac{\pi}{\tau} k \zeta}\right\rangle=\frac{1}{\sqrt{2 \tau}} \int_{-\tau}^{+\tau} \hat{f}(\zeta) e^{-i \frac{\pi}{\tau} k \zeta} d \zeta=\sqrt{\frac{\pi}{\tau}} f\left(-\frac{k \pi}{\tau}\right)
$$

so that

$$
\hat{f}(\zeta)=\frac{\sqrt{\pi}}{\sqrt{2} \tau} \sum_{k=-\infty}^{+\infty} f\left(\frac{k \pi}{\tau}\right) e^{-i \frac{\pi}{\tau} k \zeta}
$$

Cotransforming this one gets the cardinal series expansion. Also,

$$
\int_{-\infty}^{+\infty}|f(t)|^{2} d t=\int_{-\tau}^{+\tau}|\hat{f}(\zeta)|^{2} d \zeta=\frac{\pi}{\tau} \sum_{k}\left|f\left(\frac{k \pi}{\tau}\right)\right|^{2} .
$$

The rate of $\frac{\tau}{\pi}$ samples for unit interval is called the Nyquist rate. It is well known that a slower rate of sampling is not possible in order to get exact reconstruction, while a higher rate (oversampling) may lead to reconstruction formulas with faster convergence. We will see below precise formulations of this fact.

It is quite natural to replace the equally spaced sequence $\left\{k \frac{\pi}{\tau}\right\}_{k \in Z}$ by a general sequence $\Lambda=\left\{t_{k}\right\}_{k=-\infty}^{+\infty}$, and ask to what extent the values $\left\{f\left(t_{k}\right)\right\}_{k=-\infty}^{+\infty}$ determine completely $f$, for every $f \in B_{\tau}^{2}$. If

$$
f \in B_{\tau}^{2}, f\left(t_{k}\right)=0 \quad \forall k \text { implies } f \equiv 0
$$

the sequence $\left\{t_{k}\right\}_{k=-\infty}^{+\infty}$ is called a sequence of uniqueness for $B_{\tau}^{2}$. Note that whenever $f, g \in B_{\tau}^{2}$ and $f\left(t_{k}\right)=g\left(t_{k}\right) \forall k$ then $f=g$.

This notion alone is not sufficient to allow errors in the sampled values $\left\{f\left(t_{k}\right)\right\}_{k=-\infty}^{+\infty}$. For that purpose the notion of stable sampling sequences is introduced: these are the sequences $\Lambda=\left\{t_{k}\right\}_{k}$ for which there exist two constants $A$, 
$B$ such that

$$
A \int_{-\infty}^{+\infty}|f(t)|^{2} d t \leq \sum_{k}\left|f\left(t_{k}\right)\right|^{2} \leq B \int_{-\infty}^{+\infty}|f(t)|^{2} d t
$$

for all $f \in B_{\tau}^{2}$. In particular, $\int|f(t)-g(t)|^{2} d t$ is comparable to $\sum_{k}\left|f\left(t_{k}\right)-g\left(t_{k}\right)\right|^{2}$. Thus, $f$ can be completely recovered, at least theoretically, from its samples $\left\{f\left(t_{k}\right)\right\}_{k}$ in a stable way, meaning that small errors in the samples will produce small errors in the reconstruction.

But, how does it work this reconstruction? This is a matter of (infinite dimensional) linear algebra, that is, Hilbert spaces. To show that it is convenient to work on the frequency side, that is in $L^{2}(-\tau, \tau)$; in terms of $g=\hat{f}$ the above inequality is written

$$
A\|g\|_{2}^{2} \leq \sum_{k}\left|\left\langle g, \frac{1}{\sqrt{2 \tau}} e^{i t_{k} \zeta}\right\rangle\right|^{2} \leq B\|g\|_{2}^{2} .
$$

A family $\left\{e_{k}\right\}_{k \in Z}$ of vectors in a Hilbert space $H$ satisfying

$$
A\|u\|^{2} \leq \sum_{k}\left|\left\langle u, e_{k}\right\rangle\right|^{2} \leq B\|u\|^{2}, \quad u \in H
$$

is called a frame. Frames were introduced in the article [8], which by this reason has been an influential paper in the last forty years. The frame condition is equivalent to the operator

$$
\begin{aligned}
T: H & \longrightarrow \ell^{2}(Z) \\
u & \longmapsto\left(\left\langle u, e_{k}\right\rangle\right)_{k}
\end{aligned}
$$

being one to one onto a closed subspace of $\ell^{2}(Z)$. This is in turn equivalent to the adjoint operator

$$
T^{*}: \ell^{2}(Z) \longrightarrow H
$$

being onto. A trivial computation shows that $T^{*}\left(\left\{c_{k}\right\}\right)=\sum_{k} c_{k} e_{k}$. Hence if $\left\{e_{k}\right\}_{k \in Z}$ is a frame, every $u \in H$ can be written

$$
u=\sum_{k} c_{k} e_{k}
$$

In general this expression is not unique, there are relations $\sum_{k} c_{k} e_{k}=0$, those $\left\{c_{k}\right\}$ in the kernel of $T^{*}$. Among all these expressions, the one minimizing $\sum_{k}\left|c_{k}\right|^{2}$ is of the form $c_{k}=\left\langle u, \tilde{e}_{k}\right\rangle$ for some family $\left\{\tilde{e}_{k}\right\}_{k}$ which turns out to be a frame as well, the dual frame. The dual frame of $\left\{\tilde{e}_{k}\right\}_{k}$ is $\left\{e_{k}\right\}_{k}$ so that the reconstruction formula for $u$ is

$$
u=\sum_{k}\left\langle u, \tilde{e}_{k}\right\rangle e_{k}=\sum_{k}\left\langle u, e_{k}\right\rangle \tilde{e}_{k}
$$


The redundancy of these expressions comes from linear relations between the vectors $e_{k}, \tilde{e}_{k}, k \in Z$, that is, from the kernel of $T^{*}$. Hence there is no redundancy as soon as $\operatorname{ker} T^{*}=\{0\}$; since $\operatorname{ker} T^{*}$ is the orthogonal of Range $T$, this leads in a natural way to the following notion: a family of vectors $\left(e_{k}\right)_{k \in Z}$ in a Hilbert space $H$ is called a Riesz-Fischer family or (a free family) if

$$
\left\langle u, e_{k}\right\rangle=a_{k}, \quad k \in Z
$$

has a solution $u \in H$ for every $\left(a_{k}\right) \in \ell^{2}(Z)$. In our setting, when translated back to $f$, where $u=\hat{f}$, for $e_{k}(\zeta)=\frac{1}{\sqrt{2 \tau}} e^{i t_{k} \zeta}$, this corresponds to the sequence $\Lambda=$ $\left\{t_{k}\right\}$ being an interpolating sequence in the sense that $f\left(t_{k}\right)=a_{k}, k \in Z$, has a solution $f \in B_{\tau}^{2}$ for every $\left(a_{k}\right) \in \ell^{2}(Z)$.

The frames with no redundancy are thus those for which $T$ is an isomorphism; this amounts to $T^{*}$ being an isomorphism, i.e. every $u$ has an unique expression $u=$ $\sum_{k} c_{k} e_{k}$ with $\|u\|_{2}^{2}$ comparable to $\sum_{k}\left|c_{k}\right|^{2}$. For this reason they are called as well Riesz bases or exact frames.

To summarize, $\Lambda$ is a sequence of stable sampling for $B_{\tau}^{2}$ iff the family of exponentials $\mathcal{E}(\Lambda)=\left\{e^{i t_{k} \zeta}\right\}_{k \in Z}$ is a frame of $L^{2}(-\tau, \tau)$, and a sequence of free interpolation iff $\mathcal{E}(\Lambda)$ is a free family; $\mathcal{E}(\Lambda)$ is a Riesz basis of $L^{2}(-\tau, \tau)$ iff $\Lambda$ is both of stable sampling and of interpolation (this is called too a complete interpolating sequence). Note that in this language, $\Lambda$ is a set of uniqueness if and only if $\mathcal{E}(\Lambda)$ spans the whole of $L^{2}(-\tau, \tau)$.

Beurling ([5]) had considered sup-norm versions of these problems, that is, replacing functions in $L^{2}(-\tau, \tau)$ by measures or distributions $\mu$ supported in $[-\tau, \tau]$ giving raise to bounded functions

$$
f(t)=\int_{-\tau}^{+\tau} e^{i t \zeta} d \mu(\zeta)
$$

with spectrum in $[-\tau, \tau]$. The corresponding space, larger than $B_{\tau}^{2}$, is called $B_{\tau}^{\infty}$, the Bernstein space. In this setting, $\Lambda$ is of stable sampling for $B_{\tau}^{\infty}$ if

$$
\sup _{t \in \mathbb{R}}|f(t)| \leq A \sup _{k}\left|f\left(t_{k}\right)\right|
$$

and interpolating if $f\left(t_{k}\right)=a_{k}, k \in Z$, has a solution for all bounded sequences $\left\{a_{k}\right\}_{k \in Z}$.

Complex analysis enters naturally into the picture because every function in $B_{\tau}^{\infty}, B_{\tau}^{2}$ is the restriction to the real line of an entire function, the Fourier-Laplace transform

$$
f(z)=\int_{-\tau}^{+\tau} e^{i z \zeta} d \mu(\zeta), \quad z \in \mathbb{C} .
$$

$\left(d \mu(\zeta)=g(\zeta) d \zeta, g \in L^{2}\right.$ in the case of $\left.B_{\tau}^{2}.\right)$

By the Paley-Wiener theorem, $B_{\tau}^{2}$ (resp. $B_{\tau}^{\infty}$ ) consists of those entire functions of exponential type at most $\tau$, i.e., $|f(z)|=O\left(e^{\tau|z|}\right)$ and such that $f_{\mid \mathbb{R}} \in$ $L^{2}(\mathbb{R}),\left(\right.$ resp. $\left.\in L^{\infty}(\mathbb{R})\right)$. 
From this point of view, the real sequence $\Lambda=\left(t_{k}\right)_{k \in Z}$ might be replaced by a general complex sequence $\Lambda \subset \mathbb{C}$, all considerations up to now remain the same. To simplify the exposition we will however limit ourselves to the case $\Lambda \subset \mathbb{R}$. This complex-analysis setting, as it is often the case, leads immediately to interesting new points of view. For instance, we mentioned that the KSW theorem is the theoretical basis to digitalize analog signals $f \in B_{\tau}^{2}$, through its samples $f(k \pi / \lambda)$; now, looking at $f$ as an entire function gives immediately a discretization of $f$ as well, because by Hadamard's factorization theorem, every entire function of exponential type is characterized up to a constant by its sequence of zeroes $\left\{z_{k}\right\}$

$$
f(z)=c e^{a z} z^{m} \prod\left(1-\frac{z}{z_{k}}\right) e^{z / z_{k}}
$$

Vitushkin has studied the properties of this digitalization process.

The notions of frames, Riesz bases and so on in $L^{2}(-\tau, \tau)$ can of course be translated to $B_{\tau}^{2}$ as well. Just note that

with

$$
f(t)=\frac{1}{\sqrt{2 \pi}} \int_{-\tau}^{+\tau} \hat{f}(\zeta) e^{i t \zeta} d \zeta=\int_{-\infty}^{+\infty} f(x) K(t, x) d x
$$

$$
K(t, x)=\frac{1}{2 \pi} \int_{-\tau}^{+\tau} e^{i \zeta(t-x)} d \zeta=\frac{1}{\pi} \frac{\sin \tau(t-x)}{\tau(t-x)} .
$$

This last expression can viewed as the inner product of $f$ with the Bergman kernel $K(t, \cdot)$. Hence, statements about $\mathcal{E}(\Lambda)$ being a Riesz basis, a frame etc. of $L^{2}(-\tau, \tau)$ are equivalent to analogous statements about the family $K(\Lambda)=$ $\left\{K\left(t_{k}, \cdot\right)\right\}_{k \in Z}$ in $B_{\tau}^{2}$.

\section{Sets of Uniqueness}

In this paragraph, $B_{\tau}$ will denote any of the spaces $B_{\tau}^{2}, B_{\tau}^{\infty}$.

Complexifying time, that is, viewing $f \in B_{\tau}$ as entire functions, it is evident that every $\Lambda$ with a finite accumulation point is of uniqueness. These are the uninteresting uniqueness sets, whence we will assume $\Lambda$ discrete. It is intuitively clear that such a $\Lambda$ must have sufficiently many points; put in another way, if $S$ is not a sequence of uniqueness, then there exists $f \in B_{\tau}, f \not \equiv 0$, such that $f_{\mid S}=0$, that is, $S$ is included in the zero set $S(f)$ of $f$ and hence it cannot have too many points. Hence the question becomes one about distributions of (real) zeros of functions in $B_{\tau}$. Since whenever $f \in B_{\tau}$ and $f(\alpha)=0$ the function $g(z)=f(z) \frac{z-\beta}{z-\alpha}$ is again in $B_{\tau}$ and $g(\beta)=0$, changing a finite number of points cannot have any effect. Altogether, this means that some asymptotic density must be "small" for sequences $S$ and "big" for sequences $\Lambda$.

It is convenient to introduce the characteristic function of $S$

$$
n_{S}(t)=\#\{S \cap(0, t]\}, t>0 ; \quad n_{S}(t)=-\#\{S \cap(t, 0]\}, t<0
$$

so that $n_{S}(b)-n_{S}(a)=\#\{S \cap(a, b]\}, n_{S}(0)=0$. 
A classical theorem of Levinson establishes that if $f \in B_{\tau}$ has real zeros $S(f)$, this sequence has a finite asymptotic density

$$
D(S(f))=\lim _{|t| \rightarrow \infty} \frac{n_{S(f)}(t)}{t} \leq \frac{\tau}{\pi} .
$$

This leads in a natural way to define the Polya maximal density of a given discrete sequence $S \subset \mathbb{R}$ as

$$
D^{*}(S)=\inf \{\rho: \text { there exists a sequence } T \supset S \text { with density } \rho\}
$$

which can be defined as well only in terms of $S$ as

$$
D^{*}(S)=\lim _{\substack{\zeta \rightarrow 1 \\ \zeta<1}} \limsup _{r \rightarrow+\infty} \frac{n_{S}(r)-n_{S}(\zeta r)}{(1-\zeta) r}
$$

Obviously, $D^{*}(S) \geq \bar{D}(S)=\limsup _{|t| \rightarrow+\infty} \frac{n_{S}(t)}{t}$, the real upper density, but $D^{*}(S)$ might be strictly bigger than $\bar{D}(S)$. For instance, if say $S$ consists of the integers between $3^{k}$ and $3^{k}+3^{k-1}, k \in Z$, then $D^{*}(S)=1$, but $D(S)=\bar{D}(S)=1 / 4$, so $D^{*}(S)$ takes care of holes.

Levinson's theorem gives then that $\Lambda$ is a set of uniqueness for $B_{\tau}$ whenever $D^{*}(\Lambda)>\frac{\tau}{\pi}$. To obtain a sharp result one needs to introduce another density, and this is what Beurling and Malliavin did in the sixties in one of the finest works in the field. There are different equivalent definitions of the Beurling-Malliavin effective density; the most intuitive one uses the Rising-Sun construction of F. Riesz. Assuming for simplicity $\Lambda \subset \mathbb{R}^{+}$we look at the graphic of the step function $n_{\Lambda}(t)$

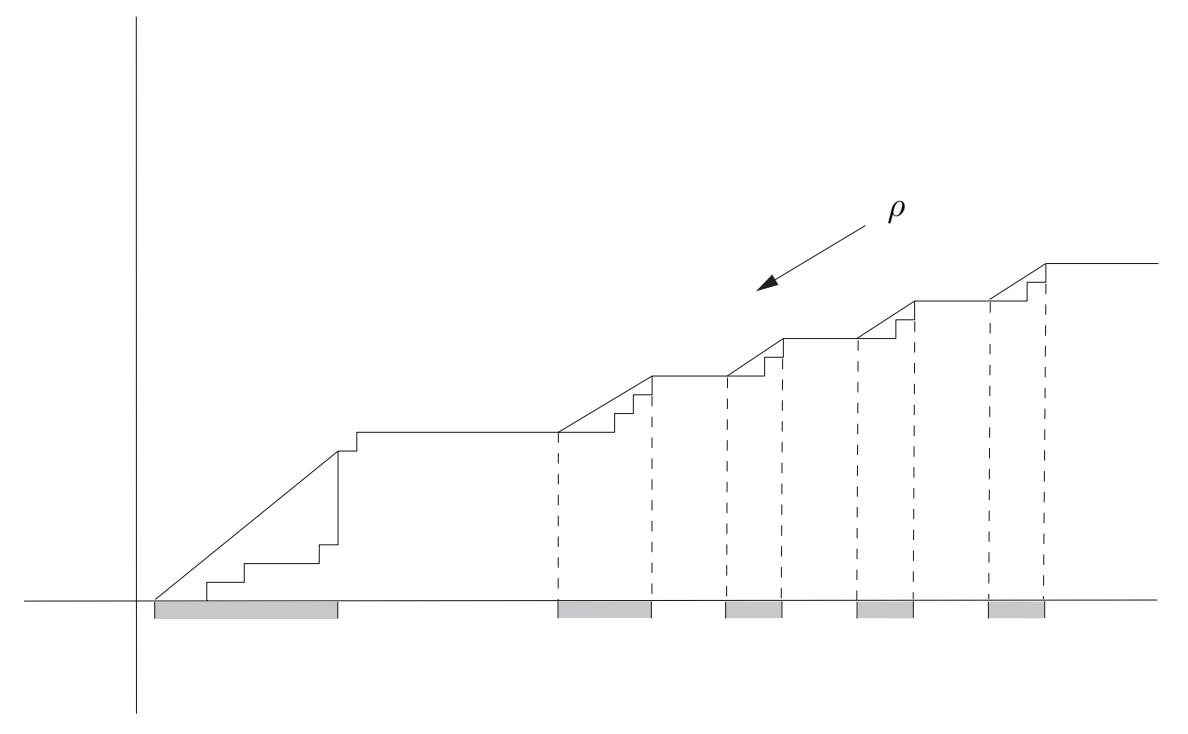


For a given $\rho$ we look at the connected components $\left(a_{k}, b_{k}\right)$ of the set $\left\{t>0: n_{\Lambda}(s)-n_{\Lambda}(t)>\rho(s-t)\right.$ for some $\left.s>t\right\}$. These are the intervals remaining in shadow when light rays with step $\rho$ illuminate the stair. Note that this set is $(0,+\infty)$ if $\rho<\bar{D}(\Lambda)$; if $\rho>\bar{D}(\Lambda)$ all components have finite length, whereas if $\rho=\bar{D}(\Lambda)$ one unbounded component may appear. The BM-effective density is defined as

$$
\tilde{D}(\Lambda)=\inf \left\{\rho: \sum_{k} \frac{\left(b_{k}-a_{k}\right)^{2}}{a_{k}^{2}}<+\infty\right\} .
$$

For a general discrete $\Lambda \subset \mathbb{R}, \tilde{D}(\Lambda)$ is defined as the maximum of $\tilde{D}\left(\Lambda \cap \mathbb{R}^{+}\right)$and $\tilde{D}\left(-\left(\Lambda \cap \mathbb{R}^{-}\right)\right)$.

In their celebrated work, Beurling and Malliavin improved Levinson's result showing that $\tilde{D}(\Lambda)>\tau / \pi$ implies $\Lambda$ being a sequence of uniqueness for $B_{\tau}$. More than that, they showed that this is sharp in the following sense: if $\Lambda$ is of uniqueness for $B_{\tau}$, then $\tilde{D}(\Lambda) \geq \tau / \pi$. Taken together, both results imply that

$$
\pi \tilde{D}(\Lambda)=\sup \left\{\tau: \Lambda \text { is a sequence of uniqueness for } B_{\tau}^{2}\right\}
$$

or which is the same

$$
\pi \tilde{D}(\Lambda)=\sup \left\{\tau: \mathcal{E}(\Lambda) \operatorname{spans} L^{2}(-\tau, \tau)\right\}
$$

The right-hand side is called the radius of completeness of the family $\mathcal{E}(\Lambda)$ and it is easily seen to be independent of the metric chosen among functions in $[-\tau, \tau]$.

In spite of the Beurling-Malliavin theorem, the precise characterization of the uniqueness sequences for $B_{\tau}^{2}$ remains, as far as I know, an open problem. A good reference for the Beurling-Malliavin results is [12].

\section{Stable Sampling and Interpolation in Dimension One}

A. Beurling characterized stable sampling and interpolating sequences in the supnorm case, for $B_{\tau}^{\infty}$, using complex analysis methods ([5]); he introduced the upper and lower uniform densities of a separated sequence $\Lambda=\left\{t_{k}\right\}_{k=-\infty}^{k \pm+\infty}$ (meaning that $\left.\inf _{k \neq \ell}\left|t_{k}-t_{\ell}\right|>0\right)$ as

$$
D_{u}^{+}(\Lambda)=\lim _{r \rightarrow \infty} \frac{n^{+}(r)}{r}, \quad D_{u}^{-}(\Lambda)=\lim _{r \rightarrow \infty} \frac{n^{-}(r)}{r}
$$

where $n^{+}(r)$ (resp. $n^{-}(r)$ ) denotes the maximum (resp. minimum) number of points of $\Lambda$ to be found in an interval of length $r$. Note that $\tilde{D}(\Lambda) \geq D_{u}^{-}(\Lambda)$. Beurling proved that $\Lambda \subset \mathbb{R}$ is of stable sampling for $B_{\tau}^{\infty}$ if and only if it contains a separated sequence $\Lambda_{0}$ with $D_{u}^{-}\left(\Lambda_{0}\right)>\frac{\tau}{\pi}$ and $\Lambda \subset \mathbb{R}$ is interpolating for $B_{\tau}^{\infty}$ if and only if it is separated and $D_{u}^{+}(\Lambda)<\frac{\tau}{\pi}$. In particular, no stable sampling interpolating sequences exist in the sup-norm case. Beurling results had been recently extended to arbitrary sequences $\Lambda \subset \mathbb{C}$ in [24]. 
In the $L^{2}$-case $B_{\tau}^{2}$, it is not hard to see that an interpolating sequence must be separated, and, on the other hand every stable sampling sequence contains a separated stable sampling subsequence. Thus one can restrict attention to separated sequences. For separated sequences Beurling results for $B_{\tau}^{\infty}$ imply in a trivial way that

$$
\begin{aligned}
& D_{u}^{-}(\Lambda)>\tau / \pi \Rightarrow \Lambda \text { stable sampling } \Rightarrow D_{u}^{-}(\Lambda) \geq \tau / \pi \\
& D_{u}^{+}(\Lambda)<\tau / \pi \Rightarrow \Lambda \text { free interpolation } \Rightarrow D_{u}^{+}(\Lambda) \leq \tau / \pi
\end{aligned}
$$

and hence it is clear that the sequences of stable sampling with no redundancy $(\mathcal{E}(\Lambda)$ Riesz basis) must have uniform density

$$
D(\Lambda)=\lim _{r \rightarrow \infty} \frac{\#\{\Lambda \cap[x, x+r]\}}{r}=\frac{\tau}{\pi}
$$

meaning that for large $r$, every integral of length $r$ must have $\frac{\tau}{\pi} r+o(r)$ points of $\Lambda$. Of course, the prototype is the sequence $Z \frac{\pi}{\tau}$.

The description of the stable sampling no redundant sequences of $B_{\pi}^{2}$ (that is the Riesz basis of $L^{2}(-\pi, \pi)$ of type $\left.\mathcal{E}(\Lambda)\right)$ was for a while a very central problem, and was solved by the Russian school $([26,22,21])$. On the way a number of perturbative results appeared among which Kadec's 1/4-theorem is the most wellknown: if $\left|t_{k}-k\right| \leq r<1 / 4, \Lambda=\left\{t_{k}\right\}$ is such a sequence.

Every such sequence $\Lambda$ gives raise to a series analogous to the cardinal series. Indeed, (assuming $\lambda_{0}=0$ in case $0 \in \Lambda$ )

$$
S(z)=\left(z-\lambda_{0}\right) \lim _{R \rightarrow \infty} \prod_{\substack{\left|t_{k}\right|<R \\ k \neq 0}}\left(1-\frac{z}{t_{k}}\right)
$$

defines the so called generating function of $\Lambda$ and the reconstruction from $a_{k}=$ $f\left(t_{k}\right)$ is given by

$$
f(t)=\sum_{k \in Z} a_{k} \frac{S(t)}{S^{\prime}\left(t_{k}\right)\left(t-t_{k}\right)} .
$$

There are different formulations of Pavlov's theorem none of which is easy, as they are all related to BMO functions or $A_{2}$ weights. We record one of them for completeness (see [11]): $\mathcal{E}(\Lambda)$ is a Riesz basis for $B_{\pi}^{2}$ if and only if $S$ has a exponential type $\pi$ and $\frac{|S(x)|^{2}}{d(x, \Lambda)^{2}}$ satisfies the Muckenhoupt $A_{2}$-condition:

"There exists a constant $C$ such that

$$
\left(\int_{I} \frac{|S(x)|^{2}}{d(x, \Lambda)^{2}} d x\right)\left(\int_{I} \frac{d(x, \Lambda)^{2}}{|S(x)|^{2}} d x\right) \leq C \quad \forall \text { interval } I . "
$$

In spite of all this progress, the description of stable sampling (separated) sequences for $B_{\tau}^{2}$ has been an open and subtle question till very recently. Indeed, it has been known for a long time that the stable sampling sequences $\Lambda$ with 
$D_{u}^{-}(\Lambda)>\frac{\tau}{\pi}$ contain a stable sampling and non redundant (interpolating) subsequence, but also examples of stable sampling sequences with no such subsequences are known ([32]).

A very important breakthrough has been recently done in [25]. Ortega-Cerdà and Seip succeed, by bringing into the picture the de Branges's theory of Hilbert spaces of entire functions, to give a complete characterization of the stable sampling sequences for the Paley-Wiener space $B_{\pi}^{2}$, or, which is the same thing, the Fourier frames $\mathcal{E}(\Lambda)$. This is one more example of the remarkable influence of de Branges work in modern analysis.

A de Branges space is a Hilbert space $H$ of entire functions fulfilling:

(H1) If $f \in H, \zeta \notin \mathbb{R}$ and $f(\zeta)=0$, the function $g(z)=f(z) \frac{z-\bar{\zeta}}{z-\zeta}$ is in $H$ with the same norm.

(H2) For every $\zeta \notin \mathbb{R}, f \mapsto f(\zeta)$ is a continuous functional.

(H3) If $f \in H$, the function $f^{*}(z)=\overline{f(\bar{z})}$ is also in $H$ with the same norm.

A fundamental theorem of de Branges ([6]) states that such a space $H$ is of the following form: there exists a function $E$ in the Hermite-Biehler class $H B$ (entire functions with no zeros in the upper half plane and such that $|E(z)| \geq|E(\bar{z})|$ for $\operatorname{Im} z>0)$ and $H$ consists of the entire functions $f$ such that

$$
\sup _{y} \int_{-\infty}^{+\infty} \frac{|f(x+i y)|^{2}}{\mid E\left(x+\left.i(y)\right|^{2}\right.} d y<+\infty
$$

the norm of $f$ being $\|f\|_{E}^{2}=\int_{-\infty}^{+\infty} \frac{|f(x)|^{2}}{|E(x)|^{2}} d x$. The Paley-Wiener space $B_{\pi}^{2}$ corresponds to $E(z)=e^{-i \pi z}$.

With these definitions, the result by Ortega-Cerdà and Seip is stated as follows: a separated sequence $\Lambda$ of real numbers is stable sampling for $B_{\pi}^{2}$ if and only if there exist two entire functions $E, F \in H B$ such that $H(E)=B_{\pi}^{2}$ and $\Lambda$ is the zero-sequence of $E F+E^{*} F^{*}$.

The functions $E \in H B$ such that $H(E)=B_{\pi}^{2}$ have been described in [18]. An obvious comment about this theorem is that the description does not involve $\Lambda$ itself alone; such a description is probably not possible with a simple formulation. In spite of this, Ortega-Cerdà and Seip are able to draw some interesting applications of the theorem. For instance, they show that there exists a complete interpolating sequence $\Gamma=\left\{\gamma_{k}\right\}_{k \in Z}$ such that for every $k \in Z$ there is at least one $t \in \Lambda$ with $\gamma_{k} \leq t<\gamma_{k+1}$, that is, a stable sampling sequence is always denser than some complete interpolating sequence. Another interesting feature that they show is the following: if $\Lambda$ is a stable sampling sequence for $B_{\pi}^{2}$ there is another Hilbert space $H$ of entire functions, bigger than $B_{\pi}^{2}$, such that $\Lambda$ becomes a complete interpolating sequence for $H$ in the appropriate sense.

As far as I know, no analogous progress has been made in the problem of describing the interpolating sequences for $B_{\pi}^{2}$.

In connection with signal analysis applications to band-limited functions the following problem might be interesting: for which sequences of measures $\left\{\mu_{k}\right\}_{k \in Z}$, 
say with disjoint supports, does it hold

$$
A \int_{-\infty}^{+\infty}|f(t)|^{2} d t \leq \sum_{k}\left|\int_{-\infty}^{+\infty} f d \mu_{k}\right|^{2} \leq B \int_{-\infty}^{+\infty}|f(t)|^{2} d t, \quad f \in B_{\pi}^{2}
$$

for some constants $A, B$ ? The case in which the $\mu_{k}$ are the Lebesgue measures of a sequence of disjoint intervals should of course be studied first.

To close this section we briefly consider now multiband signals. This means functions in $L^{2}(\mathbb{R})$ whose spectrum is contained in a set $S$ consisting of a finite number of disjoint intervals $I_{1}, \ldots, I_{m}$, which we call $B_{S}^{2}$. Here, no easy description of $B_{S}^{2}$ as a space of entire functions is available, and no complex analysis methods work. Using operator-theory methods, Landau ([15]) was able to prove that whenever $\Lambda$ is of stable sampling for $B_{S}^{2}$,

$$
n^{-}(r) \geq \frac{m(S)}{2 \pi} r-A \log ^{+} r-B
$$

for some constants $A, B$, whereas

$$
n^{+}(r) \leq \frac{m(S)}{2 \pi} r+A \log ^{+} r+B
$$

in case $\Lambda$ is of free interpolation. In particular, one has that $D_{u}^{-}(\Lambda) \geq \frac{m(S)}{2 \pi}$, $D_{u}^{+}(\Lambda) \leq \frac{m(S)}{2 \pi}$ are necessary conditions for sampling and interpolation, respectively, in $B_{S}^{2}$. It must be pointed out that in the multiband case the BeurlingLandau conditions cannot provide a complete solution to these problems; arithmetic relations among the points of $\Lambda$ then play an important role and no density condition seems appropriate. Indeed, for instance Landau [14] constructed a symmetric sequence $\Lambda$ arbitrarily close to the integers for which $\mathcal{E}(\Lambda)$ is complete in $L^{2}(S)$, where $S$ is any finite union of the intervals $|x-2 \pi n|<\pi-\delta$, with arbitrarily large measure. As far as I know the following basic question is still unanswered.

Question. Does there at all exist, for every finite union $S=I_{1} \cup \cdots \cup I_{m}$ of finite intervals, a real sequence $\Lambda$ such that $\mathcal{E}(\Lambda)$ is a Riesz basis in $L^{2}(S)$ ?

It is known that there exist complex sequences $\Lambda$ lying in horizontal strips such that $\mathcal{E}(\Lambda)$ is a Riesz basis. The answer to the question is yes if the lengths of the intervals $I_{i}$ are commesurable, and also for two intervals ([17]). The question is essentially a trivial one in case $S$ is a convenient explosion of an interval of the same length $|S|$, as pointed out in general in the next section.

\section{Uniqueness and Stable Sampling for Multidimensional Signals}

Let us replace the interval $[-\tau, \tau]$ by a general measurable set $S$ in $\mathbb{R}^{n}$, and let $B_{S}^{2}$ denote the closed subspace of $L^{2}\left(\mathbb{R}^{n}\right)$ consisting of functions whose Fourier transform $\hat{f}(\zeta)=(2 \pi)^{-n / 2} \int_{\mathbb{R}^{n}} f(t) e^{-i t \cdot \zeta} d t$ is supported on $S$. The problem is: what can be said about uniqueness sequences, stable sampling sequences, interpolating sequences, complete interpolating sequences $\Lambda=\left\{t_{k}\right\}_{k \in Z}$ for $B_{S}^{2}$ ? As before, these 
notions correspond to the family of exponentials $\mathcal{E}(\Lambda)=\left\{e^{i t_{k} \cdot \zeta}\right\}_{k \in Z}$, where now $t_{k} \cdot \zeta$ denotes the inner product in $\mathbb{R}^{n}$, being complete, a frame, free or a Riesz bases, respectively, of $L^{2}(S)$.

There are of course certain trivial cases that can be treated just making "direct product" of the one-variable results: if $S$ is a rectangle $\left|\zeta_{i}\right| \leq \tau_{i}, i=$ $1, \ldots, n$, and $\Lambda_{i} \subset \mathbb{R}$ is a uniqueness sequence for $B_{\tau_{i}}^{2}$ (that is $\mathcal{E}\left(\Lambda_{i}\right)$ complete in $\left.L^{2}\left(-\tau_{i}, \tau_{i}\right)\right)$ it is immediate that $\Lambda_{1} \times \cdots \times \Lambda_{n}$ is a uniqueness sequence for $B_{S}^{2}$, and an analogous fact occurs with frames or Riesz basis. It is also easy to produce examples for some special sets $S$. We show this for the case of orthonormal basis; from the proof of the KSW theorem indicated at the beginning, it is clear that one will have a "cardinal series" development for functions in $B_{S}^{2}$ as soon as the exponentials $\left\{e^{i k \cdot \zeta}\right\}_{k \in Z^{n}}$ constitute an orthonormal basis of $L^{2}(S)$. This is of course the case for $S_{0}=[-\pi, \pi]^{n}$ but also for $L^{2}(S)$ if $S$ is what results if we break $S_{0}$ into a finite number of pieces and move each piece by an integer vector keeping them disjoint. Moreover, exploiting the nice behaviour of the Fourier transform under the action of the linear group, we may replace $S_{0}$ by $U S_{0}$ and $Z^{n}$ by $\left(U^{T}\right)^{-1} Z^{n}=\Lambda$. In this way one can obtain an orthonormal basis of exponentials for $L^{2}(S)$ and a cardinal series development for functions in $B_{S}^{2}$ whenever $S$ is a fundamental region of a translation group.

In this connection there is an interesting conjecture by Fuglede [9]: $L^{2}(S)$ admits an orthonormal basis of exponentials $\mathcal{E}(\Lambda)$ if and only if $S$ tiles $\mathbb{R}^{n}$ by translations, in the sense that there exists a discrete set $T \subset \mathbb{R}^{n}$ such that up to sets of measure 0 , the sets $S+t, t \in T$ are disjoint and fill $\mathbb{R}^{n}$. Fuglede himself proves the conjecture if either $\Lambda$ of $T$ is a lattice. The convex sets $S$ tiling $\mathbb{R}^{n}$ by translations have been completely characterized ([20]); in dimension $n=2$, they are exactly the symmetric polygons of 4 or 6 sides. Moreover, in this case, $T$ may be chosen to be a lattice, and hence, by Fuglede's theorem, $L^{2}(S)$ has an orthonormal basis of exponentials. Significant progress has been made on Fuglede's conjecture in some other special cases (see [13] and the references there).

As in dimension one, functions in $B_{S}^{2}$ are restriction to $\mathbb{R}^{n}$ of certain entire functions in $\mathbb{C}^{n}$. As space of entire functions, $B_{S}^{2}$ can be described in neat terms only if $S$ is convex: in this case $f \in B_{S}^{2}$ if and only if $f \in L^{2}\left(\mathbb{R}^{n}\right)$ and $|f(x+i y)|=O\left(e^{\varphi_{S}(y)}\right), x, y \in \mathbb{R}^{n}$ where $\varphi_{S}(y)=\sup _{\zeta \in S} \zeta \cdot y$ is the support function of $S$. For instance, when $S$ is the rectangle $\left|\zeta_{i}\right| \leq \tau_{i}, i=1, \ldots, n$ we get the condition $\log |f(z)| \leq \tau_{1}\left|y_{1}\right|+\cdots+\tau_{n}\left|y_{n}\right|+c$.

Certain generalizations of Levinson theorem are known $([28,1])$. For instance, if $\Lambda$ is a separated sequence with separation constant $h=\inf _{k \neq \ell}\left\|t_{k}-t_{\ell}\right\|_{\infty}$, (where $\left.\|x\|_{\infty}=\max _{j}\left|x_{j}\right|\right)$ and $\limsup _{r \rightarrow \infty} \frac{\#\left\{\Lambda \cap T_{r}\right\}}{(2 r)^{n}}=d>0$ (here $\left.T_{r}=\left\{\zeta:\left|\zeta_{i}\right| \leq r\right\}\right)$, then $\Lambda$ is a uniqueness sequence for $B_{S}^{2}$, for every rectangle $S=\left\{\left|\zeta_{i}\right| \leq \tau_{i}\right\}$ with $\tau_{1}+\cdots+\tau_{n}<\frac{\pi^{n}}{(n-1) ! 2^{n-1}} h^{n-1} d$. Note however that this result does not cover the 
trivial case $\Lambda=Z^{n}$ for $S=T_{\pi}$. Another result of this type can be found in $[5$, page 310].

In general dimension $n$, the most important contribution remains that of Landau ([15]). He was able to generalize Beurling's necessary conditions for stable sampling and interpolation, for general $S$, using methods from operator theory. The uniform upper and lower densities $D_{u}^{ \pm}(\Lambda)$ of a separated sequence are defined in an analogous way: if $n^{+}(r), n^{-}(r)$ denote respectively the maximum and minimum number of points of $\Lambda$ to be found in a translate of $r U, U$ being the unit cube,

$$
D_{u}^{+}(\Lambda)=\limsup _{r \rightarrow \infty} \frac{n^{+}(r)}{r^{n}}, \quad D_{u}^{-}(\Lambda)=\liminf _{v \rightarrow \infty} \frac{n^{-}(r)}{r^{n}} .
$$

It may be seen that the result does not change replacing $U$ by another set of measure one. Landau's results are:

if $\Lambda$ is a sequence of stable sampling for $B_{S}^{2}, \quad$ then $D_{u}^{-}(\Lambda) \geq \frac{m(S)}{(2 \pi)^{n}}$;

if $\Lambda$ is a sequence of free interpolation, $\quad$ then $D_{u}^{+}(\Lambda) \leq \frac{m(S)}{(2 \pi)^{n}}$.

The method of proof of Landau is to give a firm-ground justification of the following, which a fortiori follows from the existence of stable sampling sequences. Suppose a rectangle $R$ fixed, it has a finite number of points of $\Lambda$ : if one thinks in functions well concentrated on $R$ it is intuitively clear from the stability of the sampling that the sampled values off $R$ must not affect too much and maybe thought as 0 . In this way they are essentially characterized by a finite number of samples, whence one concludes that the space of functions in $B_{S}^{2}$ which "leave essentially in a rectangle $R$ " has a "finite dimension". In a certain way, Landau reverses this line of reasoning. Landau's results imply that the Nyquist rate of sampling cannot be improved by any means.

Let us assume that $n=2, S=\left[-\tau_{1}, \tau_{1}\right] \times\left[-\tau_{2}, \tau_{2}\right]$, and let $\Lambda_{1}, \Lambda_{2}$ be two separated sequences in $\mathbb{R}$. Then it is not hard to see that $\Lambda=\Lambda_{1} \times \Lambda_{2}$ is of stable sampling for $B_{S}^{2}$ if and only if each $\Lambda_{i}$ is for $B_{S_{i}}^{2}$. On the other hand, $D_{u}^{-}(\Lambda)$ is at least $D_{u}^{-}\left(\Lambda_{1}\right) D_{u}^{-}\left(\Lambda_{2}\right)$, whence it may be made $\geq \frac{\tau_{1} \tau_{2}}{\pi^{2}}$ taking $D_{u}^{-}\left(\Lambda_{1}\right)$ big enough and $D_{u}^{-}\left(\Lambda_{2}\right)$ small, say $<\frac{\tau_{2}}{\pi}$. Combining both facts we see that $D_{u}^{-}(\Lambda)>\frac{m(S)}{(2 \pi)^{n}}$ cannot be a sufficient condition if $n \geq 2$. The same considerations suggests that some "directional uniform density conditions" should come into the picture in order to obtain sharp results.

Concerning Riesz basis in $B_{S}^{2}$, the following natural question seems unanswered.

Question. Does there at all exist a sequence $\Lambda \subset \mathbb{R}^{n}$ such that $\mathcal{E}(\Lambda)$ is a Riesz basis for $B_{S}^{2}$ ?

Besides the "direct product" situations and the trivial ones described before the only known result, as far as I know, is a recent one in [19]: the answer is yes if $S$ is a convex symmetric polygon. The case when $S$ is a ball seems particularly 
appealing. For this case, Beurling gave a sufficient condition for stable sampling in $B_{S}^{\infty}$ : if $S$ has radius $r$, and $\sup \operatorname{dist}(\zeta, \Lambda)<\pi / 2 r, \Lambda$ is of stable sampling for $B_{S}^{\infty}$ ([5, page 30$])$.

Let us close this section with some basic comments about entire functions in several variables. Dealing with these kind of questions, and leaving aside that only for convex $S$ we can understand $B_{S}^{2}$ as a space of entire functions in $\mathbb{C}^{n}$, the common situation one encounters is: $f \in B_{S}^{2}$ vanishes on $\Lambda \subset \mathbb{R}^{n}$. Either $f$ is given and we want to understand how small $\Lambda$ must be or else $\Lambda$ is given and we must construct $f$. In dimension one, complete interpolating sequences and also stable sampling sequences appear, as we have mentioned, as zero sequences of generating functions, and one can use infinite products to construct entire functions. Now, in dimension $n>1$, the zeros of analytic functions have complex dimension $n-1$, and one needs $n-1$ analytic functions to define, generically speaking, a discrete set. In one dimension, division of analytic functions is an easy task, not at all so in dimension $\geq 2$. All this helps explaining the difficulties in dealing with the multidimensional problem.

In several complex variables, varieties of zeros of analytic functions and interpolation problems from these varieties have of course been considered, and very intensively, in the last decades. But the motivation comes from other sources. Among them, one of the most important is of course Partial Differential Equations, and we show by an example what the connexion is. Let $P(D)$ be a partial differential operator with constant coefficients $D=\left(-i \frac{\partial}{\partial x_{1}}, \ldots,-i \frac{\partial}{\partial x_{n}}\right)$ : if $z_{0} \in \mathbb{C}^{n}$ satisfies $P\left(z_{0}\right)=0$ then a solution of $P(D) u=0$ is $u(x)=\exp \left(i x \cdot z_{0}\right)$, and also one has solutions $Q(x) e^{i x \cdot z_{0}}$ with a polynomial $Q$ depending on the multiplicity. These are called exponential-polynomial solutions. Euler proved that in dimension one, the finite linear combinations of exponential-polynomial solutions give all solutions. An $n$-dimensional version of this is the Fundamental Principle of Ehrenpreis-Palamodov: $f \in C^{\infty}\left(\mathbb{R}^{n}\right)$ is a solution of $P(D) f=0$ if and only if it can be represented as

$$
f(\tau)=\sum_{j=1}^{J} \int_{V_{j}} \partial_{j}\left(e^{i x-z}\right) d u_{j}(z)
$$

where the $V_{j}$ are algebraic varieties contained in $V=\left\{z \in \mathbb{C}^{n}: P(z)=0\right\}$, the $\partial_{j}$ are partial differential operators with constants coefficients and the $u_{j}$ are measures supported on $V_{j} . P(D) f=0$ is equivalent to $0=\left\langle f,{ }^{t} P(D) u\right\rangle$ for every distribution $u$ with compact support, whence to $f$ defining a well-defined functional on the quotient space $\mathcal{E}^{\prime}\left(\mathbb{R}^{n}\right) /{ }^{t} P(D) \mathcal{E}^{\prime}\left(\mathbb{R}^{n}\right)$ : by Fourier transform this is $\widehat{\mathcal{E}}^{\prime}\left(\mathbb{R}^{n}\right) / p \cdot \widehat{\mathcal{E}}^{\prime}$ which is a space of analytic functions on $V$. Proving the theorem amounts to understanding this quotient space, and this is about functions vanishing on $V$ and also about extending functions defined on $V$.

Incidentally, Beurling applied his condition about sampling for $B_{S}^{2}, S$ a ball, to deal with the equation $P(D) u=f$, say with $f \in L^{2}(S)$. By Fourier transform 
the solution $u$ must satisfy $P(\zeta) \hat{u}=\hat{f}$, whence $\hat{u}=\hat{f} / P$ but this may lead to problems because of the zeros of $P$. Beurling's idea is quite nice and simple: if $\Lambda$ is of stable sampling for $B_{S}^{2}$, without loosing information, the above is equivalent to $P(\zeta) \hat{u}(\zeta)=\hat{f}(\zeta)$ for $\zeta \in \Lambda$, and choosing $\Lambda$ so that $|P(\zeta)|$ is bounded below on $\Lambda$ (which is always possible) one can invert $P(D)$ in a stable way. In terms of the dual frame $\tilde{e}_{k}$ of $e^{i \lambda_{k} \zeta}$ in $L^{2}(S)$, if

$$
f(\zeta)=\sum_{k}\left\langle f, \tilde{e}_{k}\right\rangle e^{i \lambda_{k} \zeta}
$$

the solution is simply

$$
u(z)=\sum_{k} \frac{\left\langle f, \tilde{e}_{k}\right\rangle}{P\left(\lambda_{k}\right)} e^{i \lambda_{k} \zeta} .
$$

This method can be used as well to show the existence of a fundamental solution.

Note that the fundamental principle has something in common, in fact, with the questions we are discussing, namely, the issue is to represent certain functions as superpositions of complex exponentials. A general setting would be as follows: we are given $N, M$ in $\mathbb{C}^{n}$ and ask which functions on $N$ can be represented as Fourier-Laplace transforms of functions or measures on $M$

$$
f(\omega)=\int_{M} e^{i z \omega} g(z) d \sigma(z), \quad \omega \in N
$$

A fortiori, these functions must be restrictions to $N$ of entire functions but if $N$ is totally real this would imply no restriction. Usually $g$ is obtained from $f$ by some kind of Fourier-Laplace transform too, so this is, vaguely, a question about inverting Fourier-Laplace transforms. A general scheme leading to such formulas, which includes most of the known cases has been showed in [3]. Stable sampling sequences $\Lambda$ for $B_{S}^{2}$ correspond to $N=S, M=\Lambda$. In dimension $n>1$, there is more choice for the dimension of $M$, e.g. it could be of dimension $k, 0 \leq k \leq$ $n-1$. For instance, for Paley-Wiener spaces $B_{S}^{2}$ with $S \subset \mathbb{R}^{n}$ it makes sense, mathematically speaking, (maybe not so from the signal analysis point of view) to ask for "sampling manifolds" $M$ of dimension $k \geq 1$, which would correspond to "continuous frames". The point we want to emphasize is that the techniques developed in several complex variables so far are better adapted to deal with the case $k=n-1$.

\section{Time-Frequency Analysis and Gabor Wavelets}

In time-frequency analysis, the exponentials $e^{i \zeta \cdot t}$ are replaced by localized versions $G_{b, \zeta}(t)=G(t-b) e^{i \zeta t}$. Here $G$ is a window function, that is, an $L^{2}$-function with $\|G\|_{2}=1$, which we may think as being centered at 0 , and localized in time. 
The following analogous of Fourier formula holds for every $f \in L^{2}(\mathbb{R})$

$$
\begin{gathered}
f(t)=\frac{1}{2 \pi} \int_{-\infty}^{+\infty} \int_{-\infty}^{+\infty}\left\langle f, G_{b, \zeta}\right\rangle G_{b, \zeta}(t) d b d \zeta \\
\int_{-\infty}^{+\infty}|f(t)|^{2} d t=\frac{1}{2 \pi} \int_{-\infty}^{+\infty} \int_{-\infty}^{+\infty}\left|\left\langle f, G_{b, \zeta}\right\rangle\right|^{2} d b d \zeta
\end{gathered}
$$

The function $\tilde{f}(b, \zeta)=\left\langle f, G_{b, \zeta}\right\rangle$ is called the windowed Fourier transform of $f$; in view of the above $\frac{1}{2 \pi}|\tilde{f}|^{2}$ is a density energy in the time-frequency plane. In time, $G_{b, \zeta}$ is thought as centered at $b$ and with most of its energy in $[b-\sigma, b+\sigma]$, where $\sigma=\sigma(G)=\int t^{2}|G(t)|^{2} d t$; a computation shows that $\left(G_{b, \zeta}\right)^{\wedge}(w)=e^{i b(\zeta-w)} \hat{G}(w-\zeta)$; assuming $\hat{G}$ centered at 0 as well, in loose terms one can thus think in the atom $G_{b, \zeta}$ as occupying in the time-frequency plane a box centered at $(b, \zeta)$ with sides $\sigma(G)$, $\sigma(\hat{G})$ respectively. The above formulas say that $f \mapsto \tilde{f}$ is an isometry up to a constant, of $L^{2}(\mathbb{R})$ onto a closed subspace $H_{G}$ of $L^{2}\left(\mathbb{R}^{2}\right)$ and that the family $\left\{G_{b, \zeta}\right\}_{b, \zeta \in \mathbb{R}}$ is a sort of "doubling continuous" orthonormal basis in $L^{2}(\mathbb{R})$ because

$$
f=\frac{1}{2 \pi} \iint\left\langle f, G_{b, \zeta}\right\rangle G_{b, \zeta} d b d \zeta .
$$

The windowed Fourier transform $\tilde{f}$ is of interest in signal analysis because it provides local information about $f$ simultaneously in time and frequency. The "precision" of this information is measured by $\sigma$ in time and $\hat{\sigma}=\sigma(\hat{G})$ in frequency (because $\left.\tilde{f}(b, \zeta)=\left\langle f, G_{b, \zeta}\right\rangle=\left\langle\hat{f}, \hat{G}_{b, \zeta}\right\rangle\right)$. The uncertainty principle in Harmonic Analysis states that $\sigma \hat{\sigma}$ is bounded below, meaning that it is impossible to made precise both information simultaneously. It is well-known that $\sigma(G) \sigma(\hat{G})$ achieves its minimum value for Gaussian windows. The $G_{b, \zeta}$ are called Gabor atoms or Gabor wavelets.

It is intuitively clear that $\tilde{f}(b, \zeta)$ being a function of two variables, there exists redundancy in the above representation. It is therefore quite natural to try to discretize this representation and to extract redundancy. In this way we are led to the following questions: for which discrete sequences $\Lambda=\left\{\left(b_{k}, \zeta_{k}\right)\right\}_{k \in Z}$ of points in $\mathbb{R}^{2}$ the family $G(\Lambda)$ of time-frequency atoms $G_{k}=G_{b_{k}, \zeta_{k}}$ is complete, a frame, or a Riesz basis in $L^{2}(\mathbb{R})$ ? This is equivalent to the sequence $\Lambda$ being of uniqueness, of stable sampling or complete interpolating for the space $H_{G}$. For some special choices of the window function $G$ the space $H_{G}$ is isometric to a Hilbert space of entire functions. For instance, for $G(x)=\pi^{-\frac{1}{4}} e^{-\frac{x^{2}}{2}}$, one gets, with $z=b-i \zeta$

$$
\tilde{f}(b, \zeta)=e^{-\frac{i}{2} b \zeta} e^{-\frac{1}{4}|z|^{2}} B f(z)
$$

where $B f(z)$ is the entire function (Bargmann transform)

$$
B f(z)=\pi^{-\frac{1}{4}} e^{-\frac{\tau^{2}}{4}} \int_{-\infty}^{+\infty} f(t) e^{-\frac{t^{2}}{2}} e^{t z} d t
$$


The entire functions $F=B f$ arising in this way satisfy thus

$$
\int|F(z)|^{2} e^{-\frac{|z|^{2}}{2}} d A(z)<+\infty
$$

and constitute the so called Bargmann-Fock space.

For separated sequences $\Lambda=\left\{\left(b_{k}, \zeta_{k}\right)\right\}_{k \in Z}$, the densities to be taken under consideration are again the Beurling-Landau uniform densities

$$
D_{u}^{-}(\Lambda)=\liminf _{r \rightarrow \infty} \frac{n^{-}(r)}{\pi r^{2}}, \quad D_{u}^{+}(\Lambda)=\limsup _{r \rightarrow \infty} \frac{n^{+}(r)}{\pi r^{2}}
$$

where $n^{+}(r), n^{-}(r)$ respectively denote the maximum and minimum number of points of $\Lambda$ to be found in a disc of radius $r$. The Landau necessity conditions hold as well in this case for general windows $([27])$, that is, a frame $G(\Lambda)$ must satisfy $D_{u}^{-}(\Lambda) \geq \frac{1}{2 \pi}$ and a free system $G(\Lambda)$ must satisfy $D_{u}^{+}(\Lambda) \leq \frac{1}{2 \pi}$. In particular, Riesz basis $G(\Lambda)$ can occur only if $\Lambda$ has a uniform density $\frac{1}{2 \pi}$. For a regular lattice $\Lambda$ of the form $\left(n b_{0}, m \zeta_{0}\right)_{n, m \in Z}$, this amounts to $b_{0} \zeta_{0}=2 \pi$. But for regular lattices with $b_{0} \zeta_{0}=2 \pi$, the Balian-Low theorem establishes that if $G(\Lambda)$ is a frame then either $\sigma(G)=+\infty$ or $\sigma(\hat{G})=+\infty$. That is, at the critical density, one cannot have a Riesz basis with good localization properties both in time and frequency (see [7]). The situation is thus quite different than the one with band-limited functions.

I do not know whether it exists a version of the Balian-Low theorem for general (separated) sequences. It is known that for windows satisfying

$$
\int|x|^{2+\varepsilon}|g(x)|^{2} d x<+\infty, \quad \int|\zeta|^{2+\varepsilon}|\hat{g}(\zeta)|^{2} d \zeta<+\infty
$$

for some $\varepsilon>0$, no orthonormal basis of $G_{b, \zeta}$ 's exists. One might guess for instance that whenever $\sigma(G)+\sigma(\hat{G})$ is finite (or some other localization property of $G, \hat{G})$ then Landau's necessary conditions can be strengthened to strict inequalities $D_{u}^{-}(\Lambda)>\frac{1}{2 \pi}, D_{u}^{+}(\Lambda)<\frac{1}{2 \pi}$.

For gaussian windows this is indeed the case, and even the converse is true: $G(\Lambda)$ is a frame if and only if $D_{u}^{-}(\Lambda)>\frac{1}{2 \pi}$ and a free system if and only if $D_{u}^{+}(\Lambda)<\frac{1}{2 \pi}$. This has been proved by complex analysis method's, using the Bargmann-Fock model space, in [29] and [30]. Incidentally, $G(\Lambda)$ for a regular lattice with $b_{0} \zeta_{0}=2 \pi$ is an example of a complete system which is not a frame (unstable sampling). Under mild conditions on the window $G$, it is not hard to see that if $\operatorname{dist}(p, \Lambda) \leq \varepsilon=\varepsilon(G)$ for every $p \in \mathbb{R}^{2}$, then $G(\Lambda)$ is a frame for $L^{2}(\mathbb{R})$. In particular a regular lattice $\Lambda=\left(n b_{0}, m \zeta_{0}\right)_{n, m \in Z}$ works if $b_{0} \zeta_{0}$ is small enough (an obviously expected fact in view of the doubly continuous representation above).

In particular, no complete interpolating sequences exists in the BargmannFock space, no Riesz basis of Gabor wavelets exists in $L^{2}(\mathbb{R})$. This is in strong contrast with the Paley-Wiener space $B_{\pi}^{2}$ and leads naturally to the following question. 
Question. Which is the property of a Hilbert space of entire functions upon which depends the existence of complete interpolating sequences, that is, the existence of Riesz basis of Bergman kernels?

It must be pointed out that if one replaces the exponentials by suitable cosines and sines then it is possible to obtain orthonormal basis of $L^{2}(\mathbb{R})$ (Wilson and Malvar bases) with good time-frequency localization properties (see [7, Section 4.2.2] and the references there).

The results on the Bargmann-Fock space have been successfully generalized to more general spaces $F_{\phi}^{2}$ of the following type

$$
\|F\|_{\phi}^{2}=\left\|F e^{-\phi}\right\|_{2}^{2}=\int_{\mathbb{C}}|F(z)|^{2} e^{-2 \phi(z)} d A(z)<+\infty
$$

where $\phi$ is a subharmonic function in $\mathbb{C}$ satisfying $0<m \leq \Delta \phi(z) \leq M$. In this case the densities are defined as follows:

$$
\begin{aligned}
& D_{\phi}^{-}(\Lambda)=\liminf _{r \rightarrow+\infty} \inf _{z \in \mathbb{C}} \frac{\#(\Lambda \cap D(z, r))}{\int_{D(z, r)} \Delta \phi} \\
& D_{\phi}^{+}(\Lambda)=\limsup _{r \rightarrow+\infty} \sup _{z \in \mathbb{C}} \frac{\#(\Lambda \cap D(z, r))}{\int_{D(z, r)} \Delta \phi} .
\end{aligned}
$$

In this setting, a sequence $\Lambda=\left\{\gamma_{k}\right\}_{k \in Z}$ is called of stable sampling if $\|F\|_{\phi}^{2}$ is comparable to $\sum_{k}\left|F\left(\gamma_{k}\right)\right|^{2} e^{-2 \phi\left(\gamma_{k}\right)}$ and interpolating if the interpolation problem $F\left(\gamma_{k}\right)=a_{k}$ has a solution $F$ with $\|F\|_{\phi}<+\infty$ for all sequences $\left(a_{k}\right)$ such that $\sum_{k}\left|a_{k}\right|^{2} e^{-2 \phi\left(\gamma_{k}\right)}<+\infty$. In the papers $[2,23]$ it has been proved that a (separated) sequence $\Lambda$ is interpolating if and only if $D_{\phi}^{+}(\Lambda)<\frac{1}{2 \pi}$ and stable sampling if and only if $D_{\phi}^{-}(\Lambda)>\frac{1}{2 \pi}$ (the Bargmann-Fock space corresponds to $\phi(z)=\frac{|z|^{2}}{4}$ for which $\Delta \phi=1)$.

For certain window functions $G=e^{-\Psi}$ there is a variant of the Bargmann transform allowing to identify $H_{G}$ with $F_{\phi}^{2}$ for some $\phi$ depending on $\Psi$; for these cases one has thus a way to obtain strict inequalities.

The results for the spaces $F_{\phi}^{2}$ have been recently generalized to several variables in [16]. In this case $\phi$ is a 2-homogeneous, plurisubharmonic function, and the densities $D_{\phi}^{-}(\Lambda), D_{\phi}^{+}(\Lambda)$ are defined analogously replacing the discs $D(z, v)$ by balls $B(z, r)$ and $\Delta \phi$ by $4^{n}(i \partial \bar{\partial} \phi)^{n}$; the result is that a (separated) stable sampling sequence satisfies $D_{\phi}^{-}(\Lambda) \geq \frac{1}{(2 \pi)^{n} n !}$, and an interpolating sequence must be separated and satisfy $D_{\phi}^{+}(\Lambda) \leq \frac{1}{(2 \pi)^{n} n !}$ (the strict inequalities should hold too). It is worthwhile explaining the reason why $(i \partial \bar{\partial} \phi)^{n}$ appears instead of $\Delta \phi$. The simple example $\phi\left(z_{1}, z_{2}\right)=\alpha_{1}\left|z_{1}\right|^{2}+\alpha_{2}\left|z_{2}\right|^{2}$ in $\mathbb{C}^{2}$ and $\Lambda$ a lattice of the type $\Lambda=\Lambda_{1} \times \Lambda_{2}$, $\Lambda_{i}=a_{i}(Z \times i Z)$ will do; if $\Lambda$ is to be of stable sampling, then both $\Lambda_{1}$ and $\Lambda_{2}$ must be so in one variable, and hence $a_{i}^{-2}>2 \alpha_{i} / \pi$. The asymptotic number of points in 
$\Lambda$ in a big ball $B(z, r)$ is $\operatorname{vol}(B(z, r)) / a_{1}^{2} a_{2}^{2}$, which thus exceeds $\operatorname{vol}(B(z, r)) \frac{4 \alpha_{1} \alpha_{2}}{\pi^{2}}$, expression which involves $(i \partial \bar{\partial} \phi)^{n}$ rather than $\Delta \phi$.

The situation is similar to the one discussed before for the Paley-Wiener spaces in several variables and Landau's necessary conditions. Similar considerations show as well that these density necessary conditions can not be sufficient if $n>1$. It is interesting to note a (formal) connection between the two problems. The Paley-Wiener space $B_{S}^{2}$ is not a space of type $F_{\phi}^{2}$, but it is "almost it" with $\phi(z)=\varphi_{S}(\operatorname{Im} z)$. For instance, for $S=\prod_{i=1}^{n}\left[-\tau_{i}, \tau_{i}\right], \phi(z)=\sum_{i} \tau_{i}\left|\operatorname{Im} z_{i}\right|$; in the distribution sense, $4^{n}(i \partial \bar{\partial} \phi)^{n}$ equals $n ! 2^{n} \tau_{1} \tau_{2} \ldots \tau_{n} d m(x)$ where $d m$ denotes the Lebesgue measure in $\mathbb{R}^{n}$. For a real sequence $\Lambda \subset \mathbb{R}^{n}$, one has thus $D_{u}^{+}(\Lambda)=2^{n} n ! \tau_{1} \tau_{2} \ldots \tau_{n} D_{\phi}^{+}(\Lambda)$, and we see that the critical value $\frac{1}{(2 \pi)^{n} n !}$ becomes $\frac{2^{n} \tau_{1} \ldots \tau_{n}}{(2 \pi)^{n}}=\frac{m(S)}{(2 \pi)^{n}}$ as in Landau's result. This computation can be seen to hold for a general convex $S$. As with the Paley-Wiener spaces, one might guess that some condition involving "directional densities" should be necessary.

\section{Time-Scale Analysis}

In time-scale analysis, the atoms $G_{b, \zeta}$ are replaced by wavelets $\Psi_{b, a}(t)=$ $a^{-1 / 2} \Psi\left(\frac{t-b}{a}\right)$ obtained by translation and dilation of a (real) fixed function $\Psi \in$ $L^{2}(\mathbb{R})$ with $\|\Psi\|_{2}=1$ (here $b \in \mathbb{R}, a>0$ ). If $2 \pi \int_{0}^{\infty}|\hat{\Psi}(\zeta)|^{2}|\zeta|^{-1} d \zeta=c(\Psi)<+\infty$, then one has again a reconstruction formula

$$
f=\frac{1}{c(\Psi)} \int_{-\infty}^{+\infty} \int_{0}^{\infty}\left\langle f, \Psi_{b, a}\right\rangle \Psi_{b, a} \frac{d a}{a^{2}} d b
$$

with

$$
\int_{-\infty}^{+\infty}|f(t)|^{2} d t=\frac{1}{c(\Psi)} \int_{-\infty}^{+\infty} \int_{0}^{\infty}\left|\left\langle f, \Psi_{b, a}\right\rangle\right|^{2} \frac{d a}{a^{2}} d b
$$

The function $W_{\Psi} f(b, a)=\left\langle f, \Psi_{b, a}\right\rangle=\int_{-\infty}^{+\infty} f(t) \Psi_{b, a}(t) d t$ is called the (continuous) wavelet transform of $f$, and establishes an isometry from $L^{2}(\mathbb{R})$ to a closed subspace $W_{\Psi}$ of $L^{2}\left(\mathbb{R}_{+}^{2}, \frac{d a d b}{a^{2}}\right)$. Analogously as before, it is quite natural to try to discretize this representation and to extract redundancy: for which sequences $\Lambda=$ $\left\{z_{k}\right\}_{k \in Z}$ of points in the upper half space the family $W \Psi(\Lambda)$ of wavelets $\Psi_{t_{k}}$ is a frame, a Riesz (or orthonormal) basis of $L^{2}(\mathbb{R})$ ? This amounts requiring $\Lambda$ to be of stable sampling or a complete interpolating sequence for the space $W_{\Psi}$ (it is precisely in this context that frames came back after their introduction in [8]). The first historical example is the Haar basis, for which $\Psi(x)$ equals 1 in $(0,1 / 2)$, -1 in $(1 / 2,1)$ and is zero otherwise, and $\Lambda$ is the grid $\left(n 2^{m}, 2^{m}\right)_{n, m \in Z}$. Notice that a grid of type $\left(n b_{0} a_{0}^{m}, a_{0}^{m}\right)_{n, m \in Z}$ with $a_{0}>1, b_{0}>0$ is hyperbolically regular, that is, the dyadic squares $Q_{n, m}=\left\{(b, a): a_{0}^{m}<a<a_{0}^{m+1}, n b_{0} a_{0}^{m}<b<(n+1) b_{0} a_{0}^{m}\right\}$ have constant measure with respect the hyperbolically invariant measure $\frac{d a d b}{a^{2}}$ of $\mathbb{R}_{+}^{2}$ appearing above; these are the regular lattices $\Lambda\left(a_{0}, b_{0}\right)$ to be considered here. 
Under very general conditions on $\Psi$ (for instance $\Psi$ in the Schwarz class and $\left.\int \Psi(t) d t=0\right)$ it is not hard to see that if $a_{0}, b_{0}$ are small enough then $W \Psi(\Lambda)$ is a frame for the regular lattice $\Lambda=\Lambda\left(a_{0}, b_{0}\right)$ (see [7]). This holds as well for a general $\Lambda$ which is hyperbolically separated and such that $\operatorname{dist}_{H}(p, \Lambda) \leq \varepsilon=\varepsilon(\Psi)$ (hyperbolic distance) is small enough.

If $W \Psi(\Lambda)$ is an orthonormal basis for a regular lattice $\Lambda=\Lambda\left(a_{0}, b_{0}\right)$ then $c(\Psi)=b_{0} \ln a_{0}$ (see [7, p. 63]). Normalizing $c(\Psi)$, this suggests, in analogy with the Gabor wavelet case, that $b_{0} \ln a_{0}$ could maybe play the same role as $b_{0} \zeta_{0}$ and that some critical density might exist for orthonormal or Riesz bases. Pushing the analogy further one might think as well in a version of the Balian-Low theorem for wavelets, establishing for instance that no orthonormal basis $W \Psi(\Lambda)$ exist for regular $\Lambda$ and $\Psi$ well localized in time and frequency.

All this turns out to be (fortunately) false. Stromberg and Meyer constructed nice $\Psi$ 's for which the $\Psi_{n, m}(t)=2^{-m / 2} \Psi\left(2^{-m} t-n\right), n, m \in Z$ constitute an orthonormal basis of $L^{2}(\mathbb{R})$. Later, the theory of multiresolution analysis (MRA) developed by Mallat and Meyer provided a natural framework and lead to a procedure to construct plenty of such basis, even with a regular and compactly supported $\Psi$. Concerning the existence of a critical density, the Meyer wavelet is a counterexample too, for it can be proved that $W \Psi(\Lambda(2, b))$ is a Riesz basis for all $b$ close enough to 1 (see [7] for all these facts).

But Riesz basis $W \Psi(\Lambda)$ do not always exist. For some mother wavelets $\Psi$ the situation is in fact similar to the Gabor case and a critical density exists. This is so, for instance, for the Poisson wavelet $\Psi(t)$ for which $\hat{\Psi}(\zeta)=c|\zeta| e^{-|\zeta|}$; in this case the space $W_{\Psi}$ is isometric to the space of all holomorphic functions $F(z)$ of $z=b+i a$ in $\mathbb{R}_{+}^{2}$ such that

$$
\|F\|_{2}^{2}=\int_{-\infty}^{+\infty} \int_{0}^{+\infty} a|F(z)|^{2} d b d a<+\infty .
$$

This is called a (weighted) Bergman space.

In this isometry, the frames $W \Psi(\Lambda)$ correspond as usual with the stable sampling sequences, which here are those $\Lambda=\left\{z_{k}=b_{k}+i a_{k}\right\}$ for which $\|F\|_{2}^{2}$ is comparable to $\sum_{k}\left|F\left(z_{k}\right)\right|^{2} a_{k}^{3}$, and the interpolating sequences (corresponding to free systems $W \Psi(\Lambda))$ are those for which $F\left(z_{k}\right)=\lambda_{k}$ has a solution for all sequences $\left(\lambda_{k}\right)_{k}$ such that $\sum_{k}\left|\lambda_{k}\right|^{2} a_{k}^{3}<+\infty$. Seip ([31]) characterized both type of sequences in terms of Beurling-type densities. The (pseudo)hyperbolic distance between $z, w \in \mathbb{R}_{+}^{2}$ being $d_{H}(z, w)=\left|\frac{z-w}{z-\tilde{w}}\right|$, for a pseudohyperbolically separated sequence $\Lambda$, let $n(z, r)$ the number of points of $\Lambda$ in the $d_{H}$-disc centered at $z$ of radius $r$, and let $a(r)$ denote the hyperbolic area of that disc. Writing

$$
N(z, r)=\int_{0}^{r} n(z, t) d t, \quad A(r)=\int_{0}^{r} a(t) d t
$$


the densities $D_{u}^{+}(\Lambda), D_{u}^{-}(\Lambda)$ are now defined

$$
\begin{aligned}
& D_{u}^{+}(\Lambda)=\limsup _{r \rightarrow 1} \sup _{z} \frac{N(z, r)}{A(r)} \\
& D_{u}^{-}(\Lambda)=\liminf _{r \rightarrow 1} \inf _{z} \frac{N(z, r)}{A(r)} .
\end{aligned}
$$

For a regular lattice $\Lambda=\left\{n b_{0} a_{0}^{m}+i a_{0}^{m}, n, m \in Z\right\}$ it turns out that $D_{u}^{+}(\Lambda)=$ $D_{u}^{-}(\Lambda)=2 \pi / b_{0} \ln a_{0}$; Seip proved that $\Lambda$ is a stable sampling sequence if and only if $D_{u}^{-}(\Lambda)>1$, and interpolating if and only if $D_{u}^{+}(\Lambda)<1$. In particular, no wavelet bases $W \Psi(\Lambda)$ exist for the Poisson wavelet.

We will finish this section with some comments about wavelet bases in $L^{2}\left(\mathbb{R}^{n}\right)$ with $n>1$. One of the basic constructions is the "tensor product technique" leading to separable wavelet bases of $L^{2}\left(\mathbb{R}^{n}\right)$ (see [7]), but other constructions which do not single out the $n$ axis directions are possible. In the more general context, for a mother wavelet $\Psi \in L^{2}\left(\mathbb{R}^{n}\right),\|\Psi\|_{2}=1$, a family of wavelets $\left\{\Psi_{b, M}\right\}$ is obtained from $\Psi$ replacing the scale parameter $a$ in one dimension by a matrix $M$ in the linear group $G L\left(\mathbb{R}^{n}\right)$ :

$$
\Psi_{b, M}(x)=(\operatorname{det} M)^{-1 / 2} \Psi\left(M^{-1}(x-b)\right), \quad M \in G L\left(\mathbb{R}^{n}\right), b \in \mathbb{R}^{n} .
$$

The continuous wavelet transform $f \in L^{2}\left(\mathbb{R}^{n}\right)$ is then the function $W_{\Psi} f(b, M)=$ $\left\langle f, \Psi_{b, M}\right\rangle=\int_{\mathbb{R}^{n}} f(x) \Psi_{b, M}(x) d x$.

Let now $H$ be a (connected) closed subgroup of $G L\left(\mathbb{R}^{n}\right)$ and let $d \sigma(M)$ denote the left-invariant Haar measure on $H$. It is not hard to see that if $\Psi$ is $H$-admissible in the sense that

$$
\int_{H}|\hat{\Psi}(\zeta M)|^{2} d \sigma(M)=c(\Psi)<+\infty \text { independently of } \zeta
$$

then one has again a reconstruction formula

$$
f=\frac{1}{c(\Psi)} \int_{H} \int_{\mathbb{R}^{n}}\left\langle f, \Psi_{b, M}\right\rangle \Psi_{b, M} d b \frac{d \sigma(M)}{|\operatorname{det} M|} .
$$

The subgroup $H$ is called admissible if some $H$-admissible $\Psi$ exists. For instance, the whole $H=G L\left(\mathbb{R}^{n}\right)$ is not if $n>1$; in general, if $H$ is transitive acting on $\mathbb{R}^{n} \backslash\{0\}, H$ is admissible if and only if the isotropy group $S_{H}(x)=\{M: M x=x\}$ is compact for all $x \neq 0$. More generally, if $H$ has an open orbit $U$ such that $S_{H}(x)$ is compact for $x \in U, H$ is admissible (see [4] and [10]). Of course, other examples of admissible groups, with no open orbits, are known. For instance for $H=\mathbb{R} I d$, $H$-admissible functions exist (necessarily radial).

The question of discretizing the continuous representation, that is, sampling it on a discret $\Lambda \subset \mathbb{R}^{n} \times H$ is very little studied, as far as I know. When $H$ has an open orbit $U$ with $S_{H}(x)$ trivial, then $H$ must have dimension $n$, as a Lie group. For $n=2$ there are, up to conjugation, four connected subgroups of $G L\left(\mathbb{R}^{2}\right)$ of 
dimension 2: the diagonal matrices, the triangular ones, and

$$
\begin{aligned}
& H=\left\{\left(\begin{array}{cc}
a & 0 \\
b & a^{\varepsilon}
\end{array}\right), a, b \in \mathbb{R}, a \neq 0\right\} \\
& H=\left\{\left(\begin{array}{cc}
a & b \\
-b & a
\end{array}\right), a, b \in \mathbb{R}, a b \neq 0\right\} .
\end{aligned}
$$

For all of them it is possible to choose an admissible function $\Psi$ (with Fourier transform $\hat{\Psi}$ being an indicator function) and a discrete subgroup $H_{0}$ of $H$ such that the $\Psi_{b, M}$ with $M \in H_{0}$ and $b \in M\left(Z^{n}\right)$ form an orthonormal basis of $L^{2}\left(\mathbb{R}^{n}\right)$.

\section{Acknowledgements}

The author thanks Joaquim Ortega-Cerdà for his help in the preparation of this survey.

\section{References}

[1] B. Berndtsson, Zeros of analytic functions in several variables, Ark. Mat. 16 (1978), 251-262.

[2] B. Berndtsson and J. Ortega-Cerdà, On interpolation and sampling in Hilbert spaces of analytic functions, J. Reine Angew Math. 464 (1995), 109-128.

[3] B. Berndtsson, An inequality for Fourier-Laplace transforms of entire functions, and the existence of exponential frames in Fock space, J. Funct. Anal. 149 (1997), 83-101.

[4] D. Bernier and K. F. Taylor, Wavelets from square-integrable representations, Siam J. Math. Anal. 27(2) (1996), 594-608.

[5] L. Carleson, P. Malliavin, J. Neuberger and J. Wermer, editors, "The Collected Works of Arne Beurling", vol. 2, Birkhäuser, 1989, 341-365.

[6] L. de Branges, "Hilbert spaces of entire functions", Prentice Hall Inc. Englewood Cliffs, N.J., 1968.

[7] I. Daubechies, "Ten Lectures on Wavelets", SIAM, Philadelhia, Pennsylvania, 1992.

[8] R. J. Duffin and A. C. Schaeffer, A class of nonharmonic Fourier series, Trans. Amer. Math. Soc. 72 (1952), 341-366.

[9] B. Fuglede, Commuting self-adjoint partial differential operators and a group theoretic problem, J. Funct. Anal. 16 (1974), 101-121.

[10] H. Führ, Discrete and semidiscrete wavelet transforms in higher dimensions, preprint 1999.

[11] S. V. Khrushchev, N. K. Nikol'skii and B. S. Pavlov, Unconditional bases of exponentials and reproducing kernels, in: "Complex Analysis and spectral theory", Lecture Notes in Math. vol. 864, Springer-Verlag, Berlin-Heidelberg, 1981, 214-335.

[12] P. Koosis, Leçons sur le théorème de Beurling et Malliavin, Les Publications CRM, Montreal, 1996.

[13] I. Laba, Fuglede's conjecture for a union of two intervals, preprint Princeton Univ. 
[14] H. J. Landau, A sparse regular sequence of exponentials closed on large sets, Bulletin A.M.S. 70 (1964), 566-569.

[15] H. J. Landau, Necessary density conditions for sampling and interpolation of certain entire functions, Acta Math. 117 (1967), 37-52.

[16] N. Lindholm, Sampling in weighted $L^{p}$ spaces of entire functions in $\mathbb{C}^{n}$ and estimates of the Bergman kernel, preprint Göteborg University.

[17] Y. Lyubarskii and K. Seip, Sampling and interpolating sequences for multibandlimited functions and exponential bases on disconnected sets, J. of Fourier Analysis and Applications 3(5) (1997), 599-615.

[18] Y. Lyubarskii and K. Seip, Weighted Paley-Wiener spaces, preprint 1999.

[19] Y. Lyubarskii and A. Rashdovskii, Complete interpolating sequences for Fourier transforms supported by convex symmetric polygons, preprint 2000.

[20] P. McMullen, Convex bodies which tile space by translation, Mathematika 27 (1980), $113-121$.

[21] A. M. Minkin, Reflection of exponents, and unconditional bases of exponentials, St. Petersburg Math. J. 3 (1992), 1043-1068.

[22] N. K. Nikol'skii, Bases of exponentials and the values of reproducing kernels, Dokl. Akad. Nauk SSSR 252 (1980), 1316-1320; english transl. in Sov. Math. Dokl. 21 (1980).

[23] J. Ortega-Cerdà and K. Seip, Beurling-type density theorems for weighted $L^{p}$ spaces of entire functions, J. Analyse Mathematique 75 (1998), 247-266.

[24] J. Ortega-Cerdà and K. Seip, Multipliers for entire functions and an interpolation problem of Beurling, J. Funct. Anal. 162 (1999), 400-415.

[25] J. Ortega-Cerdà and K. Seip, On Fourier Frames, preprint 2000.

[26] B. S. Pavlov, Basicity of an exponential system and Muckenhoupt's condition, Dokl. Akad. Nauk SSSR 247 (1979), 37-40; english transl. in Sov. Math. Dokl. 20 (1979).

[27] J. Ramanathan and T. Steger, Incompleteness of sparse coherent states, Applied and Computational Harmonic Analysis 2 (1995), 148-153.

[28] L. I. Ronkin, On discrete uniqueness sets for entire functions of exponential type in several variables, Sib. Mat. Zh. 19(1) (1971), 142-152; english transl. in Sib. Math. J. 19 (1978), 101-108.

[29] K. Seip, Density theorems for sampling and interpolation in the Bargmann-Fock space I, J. Reine Angew Math. 429 (1992), 91-106.

[30] K. Seip and R. Wallstén, Density theorems for sampling and interpolation in the Bargmann-Fock space II, J. Reine Angew Math. 429 (1992), 107-113.

[31] K. Seip, Beurling type density theorems in the unit disk, Invent. Math. 113 (1993), 21-39.

[32] K. Seip, On the connection between exponential bases and certain related sequences in $L^{2}(-\pi, \pi)$, J. Funct. Anal. 130 (1995), 131-160.

Departament de Matemàtiques,

Universitat Autònoma de Barcelona,

08193 Bellaterra (Barcelona), Spain

E-mail address: bruna@mat.uab.es 